\title{
CARTOGRAFIA DOS PONTOS DE CULTURA NO DISTRITO FEDERAL DO BRASIL 2017: UMA FERRAMENTA PARA O PLANEJAMENTO E GESTAOO DE POLITICAS PÚBLICAS
}

\author{
Rafael Sanzio Araújo dos Anjos \\ Pamela Elizabeth Morales Arteaga \\ Richard Denis \\ Gustavo Tolentino
}

Revista

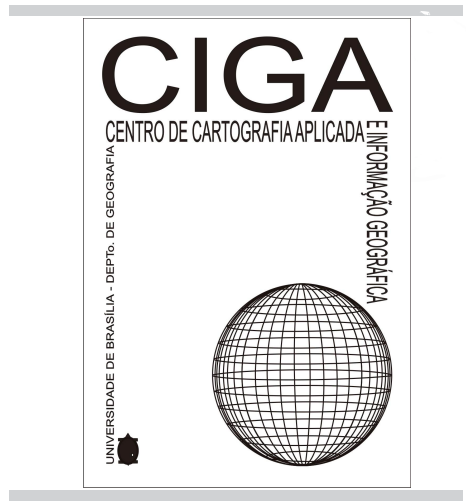

Revista Eletrônica:

Tempo - Técnica - Território, V.9, N.3 (2018), 48:56

ISSN : 2177-4366
Como citar este ar tigo:

Anjos, R.S.A.; Arteaga,P.E.M.; Denis.R.;Tolentino, G.; CARTOGRAFIA DOS PONTOS DE CULTURA NO DISTRITO FEDERAL DO BRASIL 2017: UMA FERRAMENTA PARA O PLANEJAMENTO E GESTÃO DE POLÍTICAS PÚBLICAS Revista Eletrônica: Tempo - Técnica - Território, v.9, n.3 (2018), p. 48:56 ISSN: 2177-4366.

\section{Disponível em:}

http://periodicos.unb.br/index.php/ciga/issue/view/1916

Este obra está licenciado com uma Licença Creative Commons Atribuição-NãoComercial 4.0 Internacional. 


\title{
CARTOGRAFIA DOS PONTOS DE CULTURA NO DISTRITO FEDERAL DO BRASIL 2017: UMA FERRAMENTA PARA O PLANEJAMENTO E GESTÃO DE POLÍTICAS PÚBLICAS
}

\author{
Rafael Sanzio Araújo dos Anjos (*), Pamela Elizabeth Morales Arteaga, Richard Denis, \\ Gustavo Tolentino (**) E-mail:chartografia@unb.br Site:www.ciga.unb.br
}

(*) Geógrafo, Doutor em Informações Espaciais Pós-Doutorado Cartografia Étnica. Prof. Titular da Universidade de Brasília / Diretor do Centro de Cartografia Aplicada e Informação Geográfica do Depto. de Geografia - UnB

(**) Pesquisadora Colaboradora do CIGA e Estagiários membros do Centro de pesquisa na UnB

RESUMO: O paper proposto se utiliza da linguagem cartográfica para representar espacialmente o processo de trabalho e os resultados desenvolvidos no registros de pontos de atividades culturais no Distrito Federal. Não podemos perder de vista que a informação espacial referente a forma de ocupação e uso que se processa no território constituí uma das mais estratégicas para responder sobre as inúmeras indagações do que aconteceu, do que esta acontecendo, do que pode acontecer no espaço geográfico. Utilizamos como técnicas cartográficas básicas a formação da base informacional com dados secundários e trabalho de campo nos pontos; a espacialização dos pontos de cultura em algumas Região Administrativa do DF. Referente à linguagem gráfica utilizada, usamos formas e cor para distinguir a tipologia caracterizada e registro fotográfico na legenda para aproximar o (a) usuário (a) do mundo real. Os principais resultados mostram o seguinte: primeiro, o desequilíbrio regional e quantitativo na distribuição dos pontos de cultura no DF; outro aspecto estrutural cartográfico revelado é quantidade significativa de registros concentrados nas Regiões Sdministrativas de Ceilândia, Plano Piloto, Paranoá, Planaltina e São Sebastião. Estas constatações apontam para mudanças estruturais necessárias no processo de distribuição e intervenção das ações culturais do Estado no território do DF, particularmente da Secretaria de Cultura do DGF e dos agentes culturais autônomos (individuais) e sociedades (grupo de pessoas). O projeto foi operacionalizada dentro da parceria institucional do Centro de Cartografia Aplicada e Informação Geográfica da Universidade de Brasília (CIGA-UnB) e o Instituto Terceiro Setor (ITS).

ABSTRACT: The proposed paper uses cartographic language to spatially represent the work process and the results developed in the records of points of cultural activities in the Federal District. We can not lose sight of the fact that the spatial information referring to the form of occupation and use that takes place in the territory constitutes one of the most strategic ones to answer on the countless inquiries of what happened, of what is happening, of what can happen in the geographic space. We used as basic cartographic techniques the formation of the informational base with secondary data and field work in the points; the spatialisation of the culture points in some DF Administrative Region. Regarding the graphic language used, we used shapes and color to distinguish the typology and photographic record in the legend to approximate the user (a) of the real world. The main results show the following: first, the regional and quantitative imbalance in the distribution of culture points in the DF; another cartographic structural aspect revealed is a significant amount of records concentrated in the Sdministrative Regions of Ceilândia, Piloto Plan, Paranoá, Planaltina and São Sebastião. These findings point to the necessary structural changes in the process of distribution and intervention of the State's cultural actions in the territory of the Federal District, particularly the Department of Culture of the DGF and autonomous cultural agents (individual) and societies (group of people). The project was operationalized within the institutional

Revista Eletrônica: Tempo - Técnica - Território, V.9, N.3 (2018), 48:56 ISSN: 2177-4366 
partnership of the Center for Applied Cartography and Geographic Information of the University of Brasília (CIGA-UnB) and the Third Sector Institute (ITS).

\section{1 -A CARTOGRFIA, O ORDENAMENTO DO TERRITÓRIO.}

A informação espacial referente à forma de ocupação que se processa no território constituiu uma das mais estratégicas para responder e informar sobre as inúmeras indagações do que está acontecendo, do que pode acontecer e do que aconteceu no espaço geográfico. Nesse sentido, o mapeamento das ocupações e dos espaços como ferramenta de armazenamento e comunicação de dados geográficos têm experimentado significativos avanços nas fontes geradoras das informações, principalmente nas últimas décadas, com os produtos de sensoriamento remoto de última geração e as tecnologias computacionais para manipulação e referencia mento da informação territorial.

Como ponto inicial do desenvolvimento da pesquisa, abordamos brevemente os pressupostos instrumentais e metodológicos utilizados na confecção da cartografia temática na produção do Mapa Das Culturas Populares No Distrito Federal, especificamente nas Regiões Administrativas de Taguatinga, Planaltina, Brazlândia, Paranoá, São Sebastião e Gama. Com este trabalho buscamos contribuir efetivamente para a ampliação do conhecimento sobre o espaço dessas manifestações, sobretudo no entendimento da sua organização espacial e de suporte à gestão territorial.

\section{2 - O CIGA}

Não podemos perder de vista que a Universidade de Brasília (UnB) se consolidou como uma das melhores instituições de ensino, pesquisa e extensão do Brasil, reconhecida pela qualidade e na sua produção acadêmica e pela elevada qualificação de seu corpo docente. Seguindo as suas referências básicas, o Departamento de Geografia e o Programa de Pós Graduação em Geografia da UnB constituíram, em 1999, o Centro de Cartografia Aplicada e Informação Geográfica (CIGA), com o propósito de dar maior amplitude nos projetos e parcerias em andamento, a partir da integração da educação, pesquisa e atendimento às demandas da sociedade civil. O CIGA tem uma estrutura física organizada com equipamentos básicos para o desenvolvimento de atividades direcionadas para o manuseio de ferramentas geográficas voltadas para a educação geográfica e o planejamento-gestão do território. É importante destacar que o sistema de informação territorial tem presenciado uma forte difusão de dados e tornado acessíveis outras possibilidades de representação do espaço geográfico. Por outro lado, as demandas para compreensão e resolução das complexas questões da dinâmica da sociedade são crescentes se a cartografia se confirma como uma das disciplinas melhor colocadas para responder e informar sobre o que aconteceu, o que está acontecendo de fato e o que pode acontecer no território, ou seja, as representações cartográficas possibilitam trabalhar com o passado, o presente e o futuro próximo do território. Após estes anos de atividades, o CIGA consolida a sua vocação de 
referência local, regional e nacional na produção técnica de aplicabilidades e alcance social das ferramentas geográficas ligadas à cartografia, sensoriamento remoto e sistema de informação geográficas.

\section{3 -O MAPA DAS CULTURAS POPULARES NO DISTRITO FEDERAL POR REGIÃO ADMINISTRATIVA -2017}

O CIGA realizou a primeira confecção do Mapa das Culturas Populares no Distrito Federal por Região Administrativa no primeiro semestre de 2017. Nas etapas de construção do mapa foram utilizados os seguintes processo de trabalho: Geo Serviço da Companhia de Planejamento do Distrito Federal (CODEPLAN) -GDF, equivalente ao ano de 2015, realização de um trabalho de campo onde foram obtidos as coordenas geográficas dos pontos que deu origem a tabela de coordenadas. Além das coordenadas geográficas, o trabalho de campo foi essencial para capturas de fotos dos pontos estudados. As figuras 01,02 e $\mathbf{0 3}$ mostramos processos metodológicos utilizados para confecção do mapa.

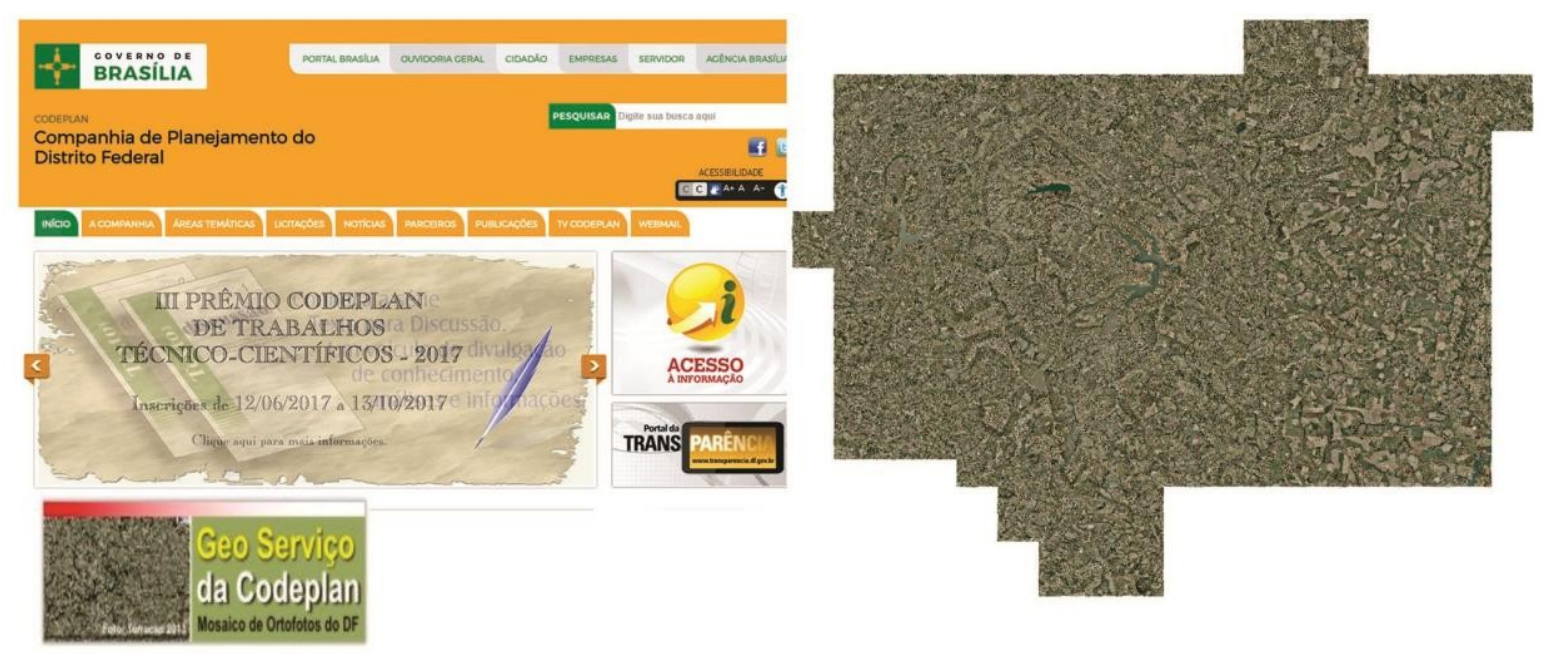

Figura 1 Fonte utilizada para a produção do mapa. 


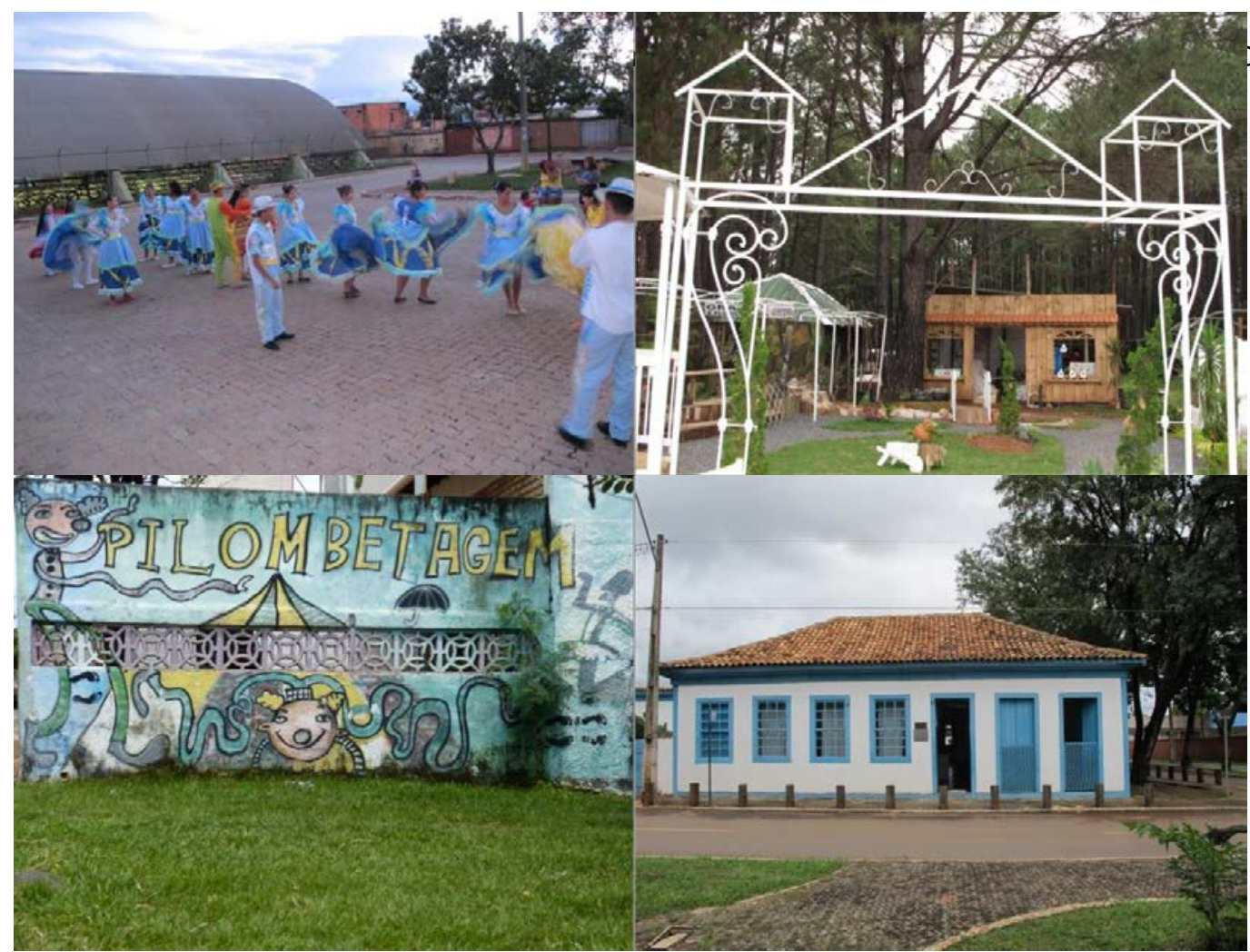

Figura 2 Fotos capturadas no trabalho de campo

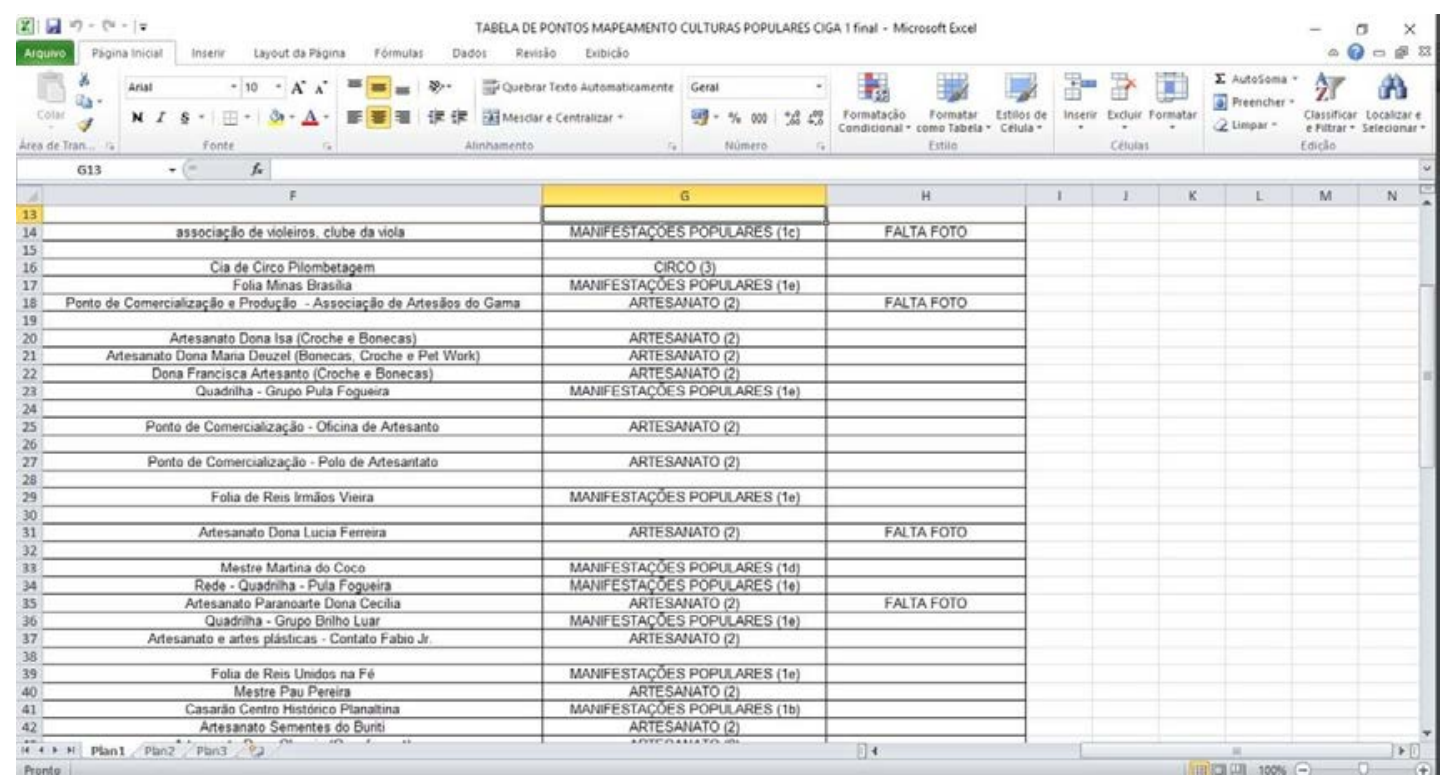

Figura 3 Tabela das coordenas geográficas originadas pelo trabalho de campo.

No processo de manipulação de dados geográficos, os softwares do QGIS Brasil, Google Earth e Corel Draw tiveram tamanha importância no desenvolvimento da atividade como localização dos pontos, transformação da base cartográfica e estruturação final do mapa. Afigura 04 mostra a superfície do software do QGIS Brasil, Google Earth e Corel Draw. 


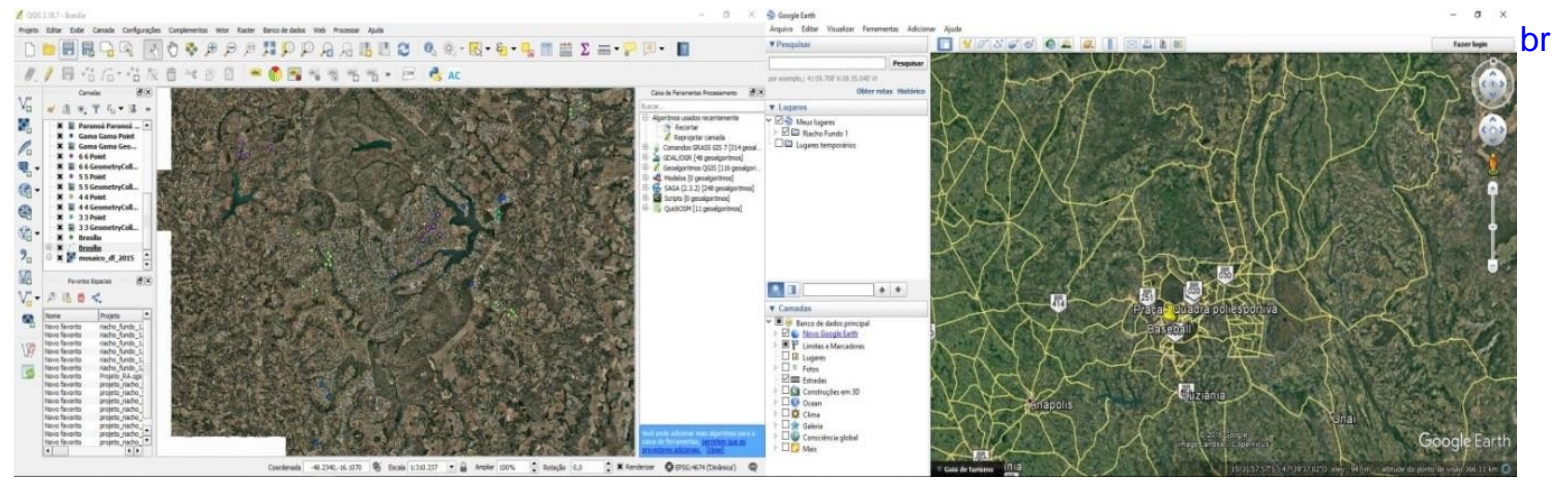

Figura 4 Software do QGIS Brasil, Google Earth e Corel Draw

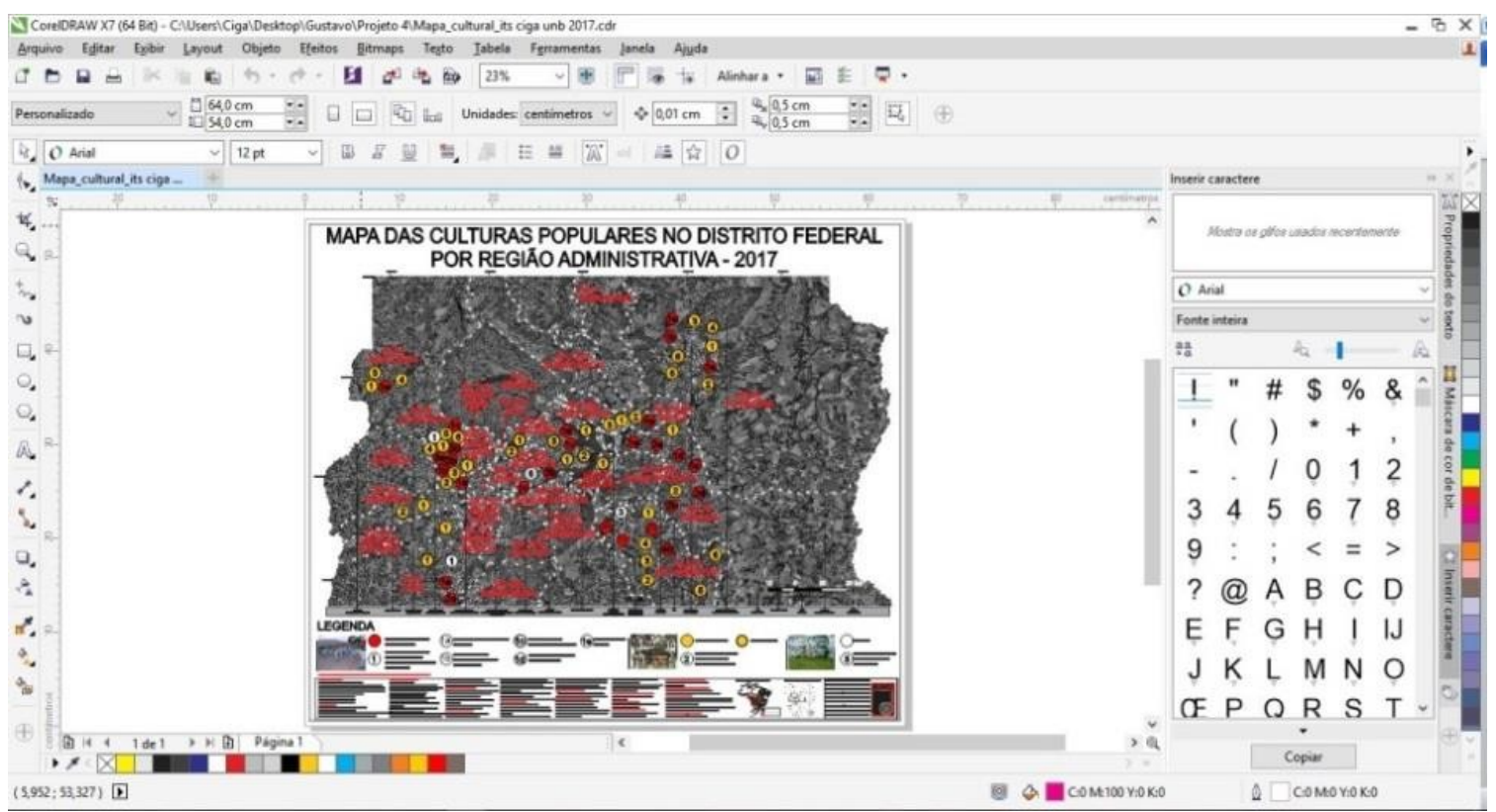

\section{4 - PRODUÇÕESCARTOGRÁFICAS}

Utilizamos a ortofoto da CODEPLAN como base territorial do Distrito Federal e o shapefile das regiões administrativa do DF (RA’S) com intuito de delimitar o território de cada cidade para fácil localização.

A aparência do território do DF foi realizada por meio do processo de recorte com utilização da ferramenta de raster do software QGIS Brasil, essa função possibilitou a visualização do aspecto da imagem de fundo do mapa. As figuras 05 e 06 mostram o recorte do território do DF pela ferramenta raster. 


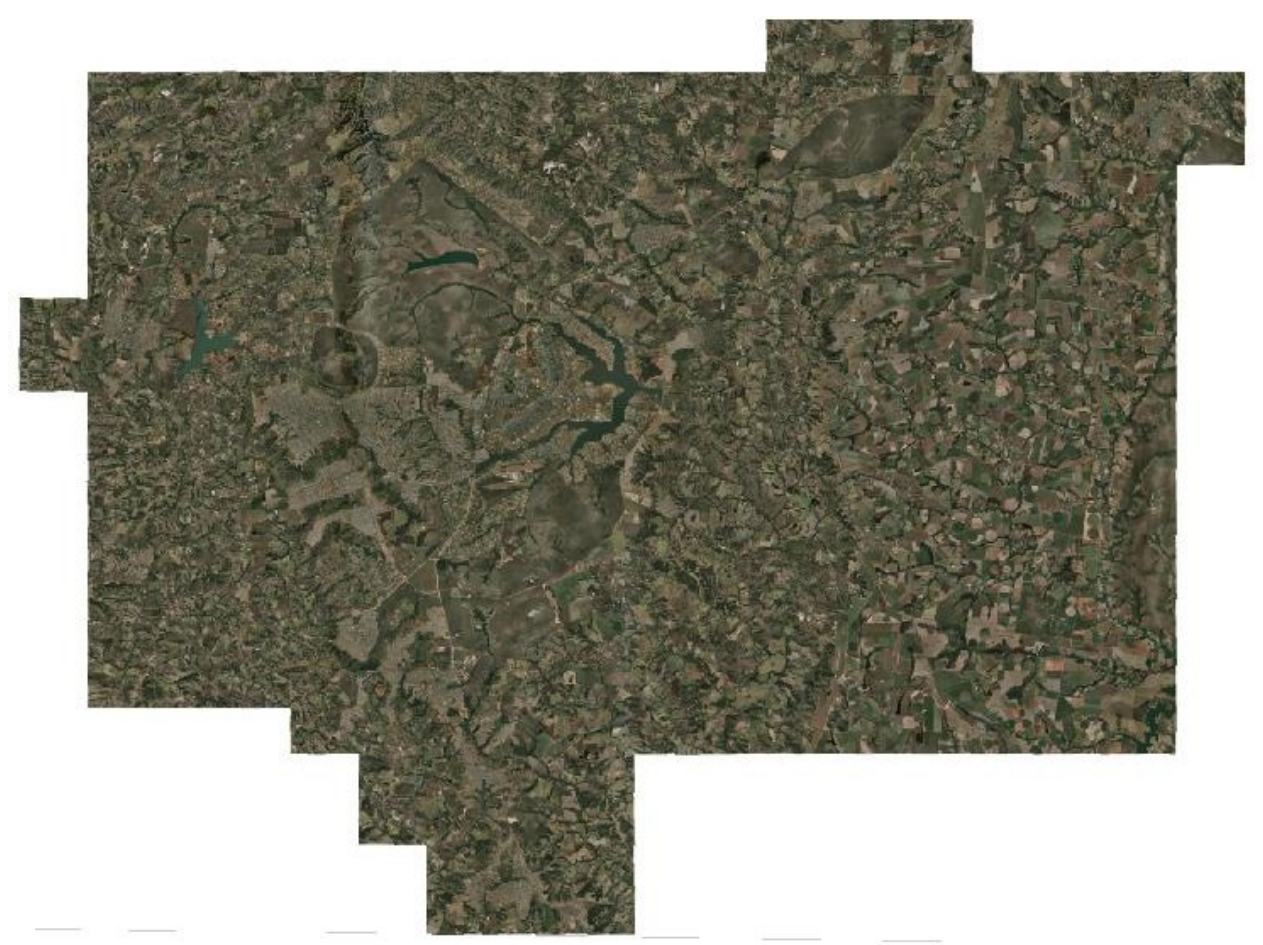

Figura 5. Ortofoto de Brasília e entorno antes do recorte.

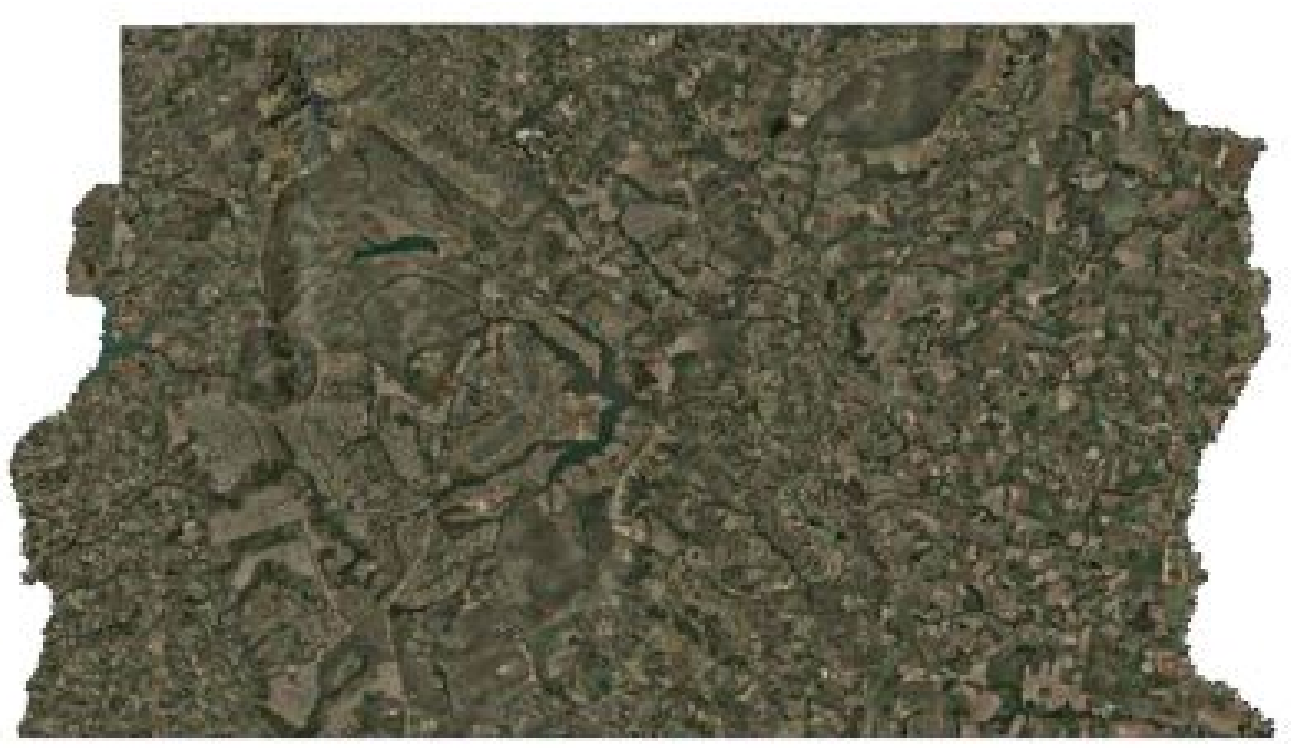

Figura 6. Recorte da delimitação do território do Distrito Federal.

A ortofoto do mapa foi manipulada para tons de cinza com intuito de dar mais destaque aos pontos de localização e os nomes das cidades como citado anteriormente. Após a alteração da cor, foi decido a utilização de uma linha tracejada branca para delimitaras RA'S, além da delimitação em branco, foram introduzidos os nomes de RA com destaque em vermelho. Afigura 07 mostra como ficou a divisão do território sobreposta na base da ortofoto da CODEPLAN. 


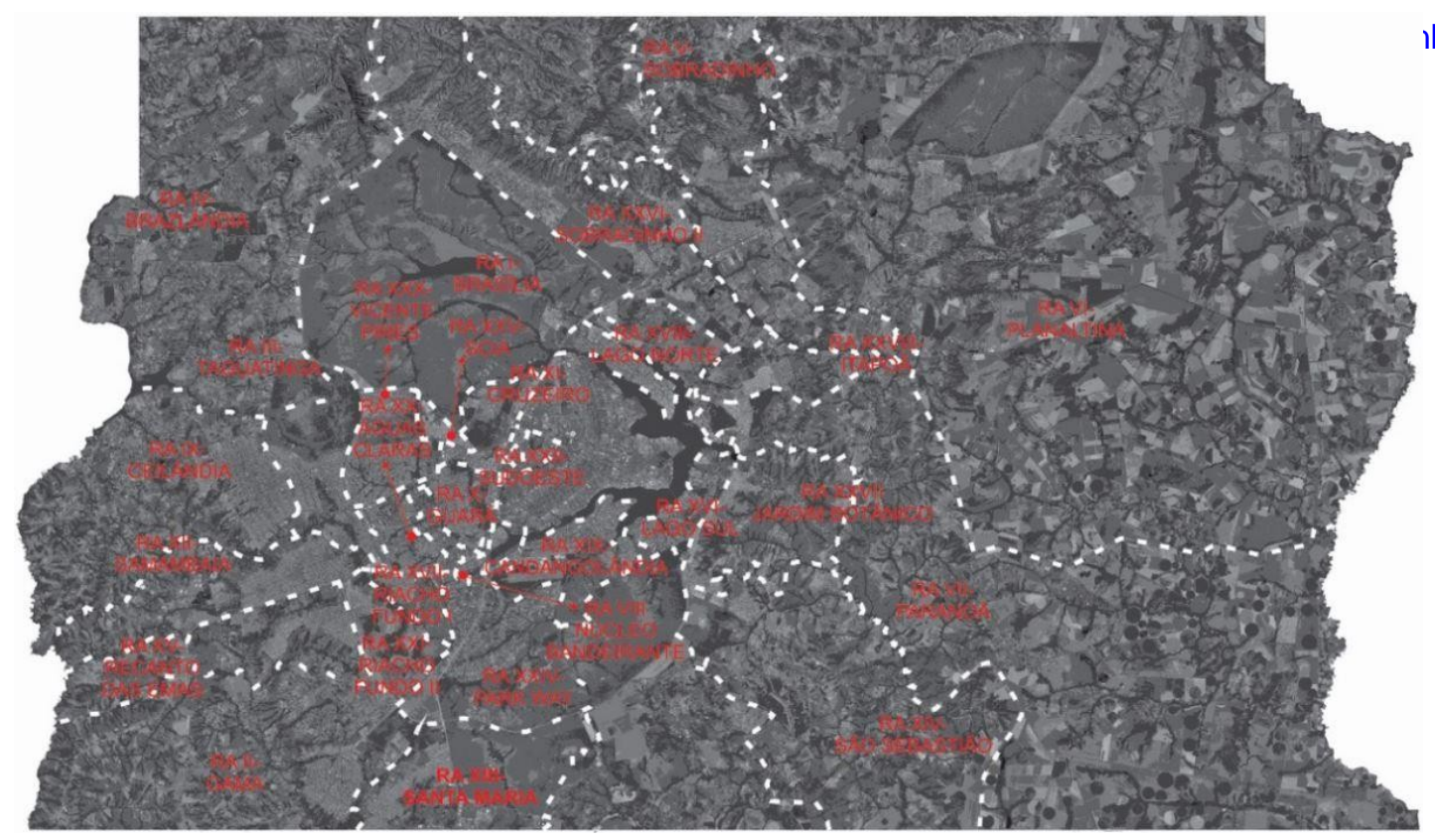

lb.br

Figura7 Plano de fundo do mapa com a utilização da ortofoto em tons de cinza e as delimitações das RA'S com nomes e linhas tracejadas.

Os pontos foram localizados pelas coordenadas geográficas no software do QGIS Brasil, sendo possível modifica-los para numeração em SGV com características distintas para melhor compreensão do leitor. O processo de localização dos pontos no território brasiliense teve como auxilio a ferramenta do software do Google Earth. Após ter toda estrutura dos pontos montados no QGIS, utilizamos o software do Corel Draw para o termino do trabalho. As figuras 08 e 09 mostram os processos de localização dos pontos obtidos no trabalho de campo. Com os pontos já alterados para SGV, o processo seguinte foi à valorização dos mesmos no território de acordo com as informações contidas.

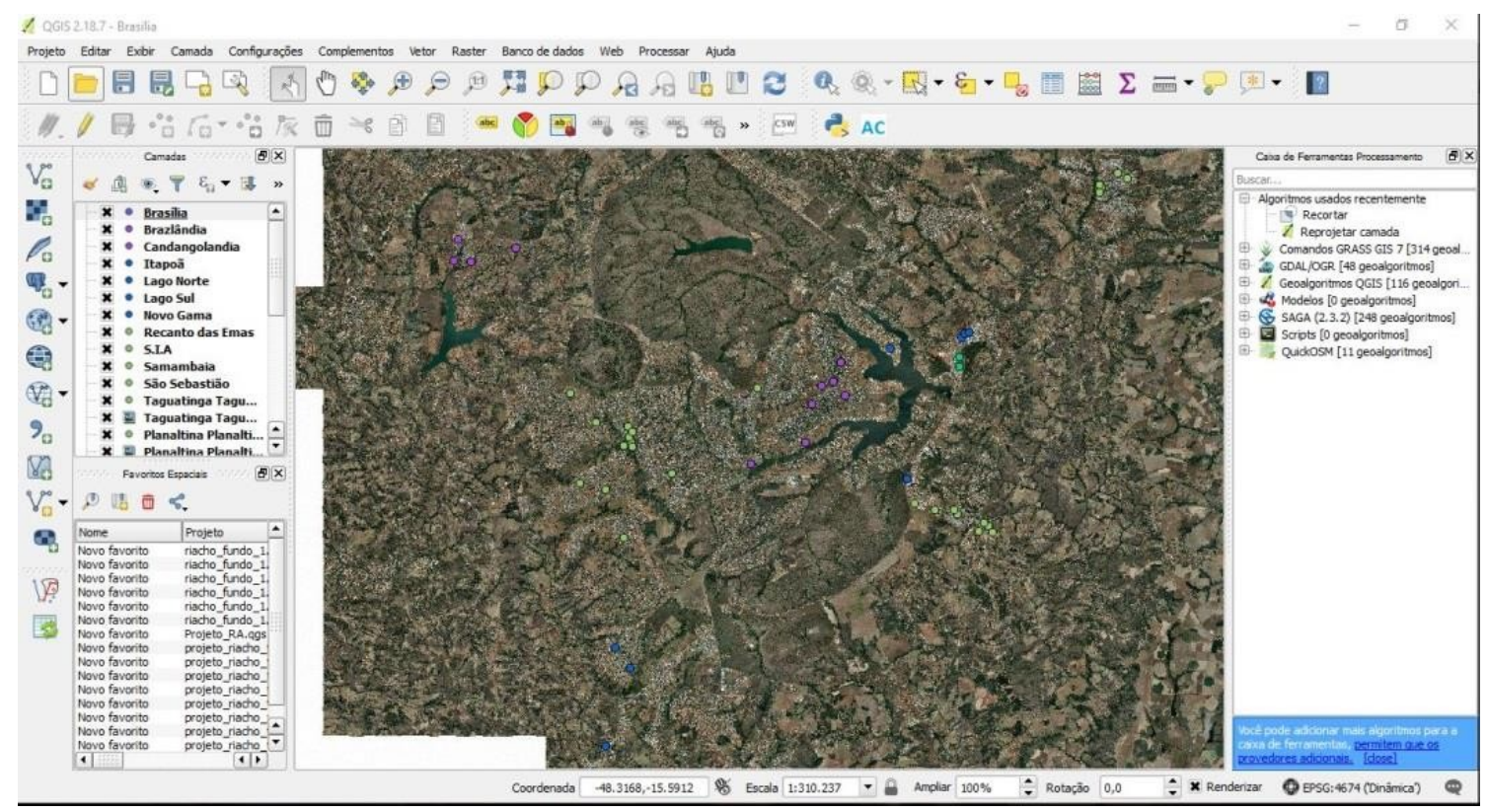

Figura 8 Manipulação das coordenas geográficas no software do QGIS Brasil.

Revista Eletrônica: Tempo - Técnica - Território, V.9, N.3 (2018), 48:56 ISSN: 2177-4366 


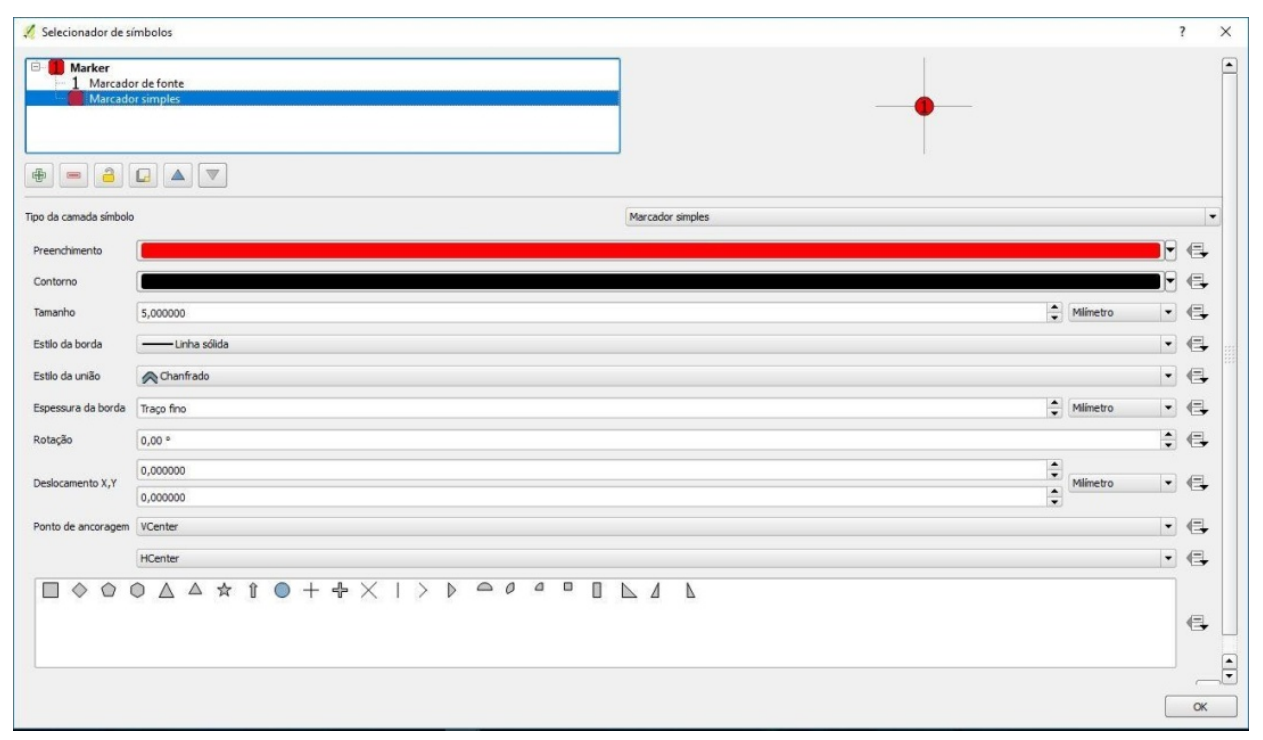

Figura 9 Manipulação dos pontos geográficos em pontos em SGV.

\section{5 - ELEMENTOS DALEGENDA}

Os elementos da legenda do mapa de culturas populares estão representados em três itens, que são: manifestações populares, categorizado com a cor vermelha, artesanato, categorizado com a cor amarela e por último os pontos de circo, categorizado com as cores brancas.

Algumas imagens foram utilizadas para ilustrar as manifestações. As imagens tiveram o papel de retratar a aparência e a estrutura de alguns pontos mapeados.

Além das categorizações e imagens, a legenda é composta por uma lista de todos os pontos mapeados de acordo com a categorização e mais dois mapas de localização do DF no Brasil e na RIDE-DF. Por último é apresentado às informações técnicas como fonte, produção e os nomes que compuseram o trabalho. A figura $\mathbf{1 0}$ apresenta a forma como os itens foram distribuídos na legenda.

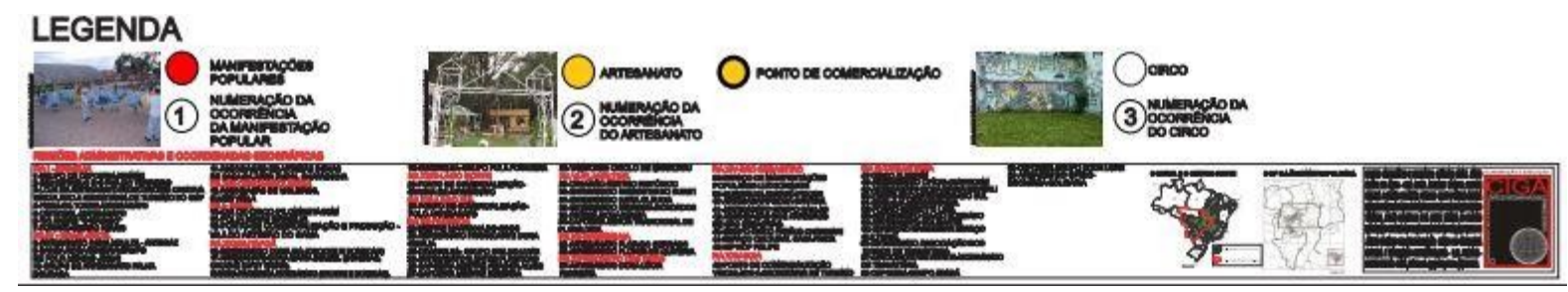

Figura 10 Categorias utilizadas para explicação da legenda.

Após o término da elaboração da legenda ocorreu uma correção de todo o layout e por fim foi concluída a aparência do mapa. A ficha técnica apresenta a última atividade decorrente do Mapa das Culturas Populares no Distrito Federal por Região Administrativa. A figura 11 apresenta a aparência final do mapa. 


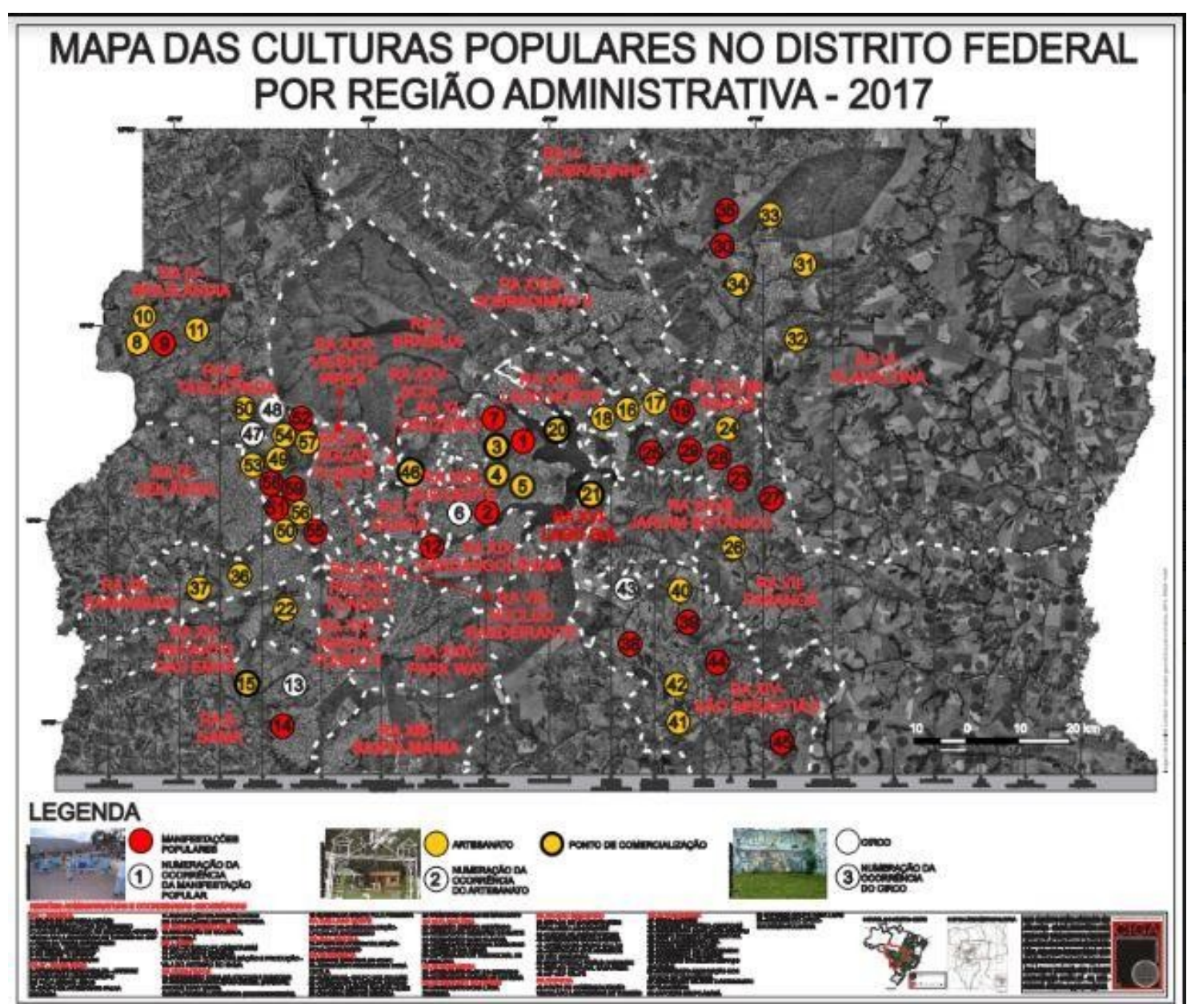

Figura 4 Aparências final do mapa.

Link do Produto Cartográfico na Parceria Realizada: http://www.rafaelsanziodosanjos.com.br/culturas.html

\section{Link do Metadado Espacial:}

http://164.41.109.9:8080/geonetwork/srv/por/catalog.search\#/metadata/d3d32fac-595c40b9-9293-8ae3ae5bd4b9

http://164.41.109.9:8080/geonetwork/srv/por/catalog.search\#/metadata/9a9757eb-bed44d3f-a957-fe006be3a818

BIBLIOGRAFIA BÁSICA:

ARTEAGA, P.M., MAKUICHI, M. F. PANTOJA, W. Retrato da Cultura Popular do DF, Brasília, Instituto Terceiro Setor-ITS. 